\title{
Bergson et les sens de la surprise : nouveauté, événement, liberté
}

\section{Arnaud Bouaniche}

\section{OpenEdition}

\section{Journals}

Édition électronique

URL : http://journals.openedition.org/alter/425

DOI : $10.4000 /$ alter.425

ISSN : 2558-7927

Éditeur :

Association ALTER, Archives Husserl (CNRS-UMR 8547)

\section{Édition imprimée}

Date de publication : 1 décembre 2016

Pagination : 83-106

ISBN : 978-2-9550449-2-6

ISSN : 1249-8947

\section{Référence électronique}

Arnaud Bouaniche, «Bergson et les sens de la surprise : nouveauté, événement, liberté », Alter [En ligne], 24 | 2016, mis en ligne le 01 décembre 2017, consulté le 20 avril 2019. URL : http:// journals.openedition.org/alter/425; DOI : 10.4000/alter.425 


\title{
BERGSON ET LES SENS DE LA SURPRISE : NOUVEAUTÉ, ÉVÉNEMENT, LIBERTÉ
}

\author{
Arnaud Bouaniche
}

L'expérience de la surprise fait l'objet d'une indéniable attention dans la philosophie contemporaine, particulièrement dans celle de la seconde moitié du $X X^{e}$ siècle, et singulièrement en France au sein d'un certain nombre de tentatives de renouvellement de la phénoménologie au contact du concept d'événement ${ }^{1}$. Au-delà de leur singularité, à la pointe d'une conception du temps où se défont les catégories du sujet et de l'objet, ces perspectives rencontrent chacune la surprise comme un fait primitif, témoin irrécusable, à même la nouveauté qui le suscite, de l'irréductible excès du réel sur la pensée. Or, la proximité est troublante, à la lecture de son œuvre, entre Bergson et ces philosophies contemporaines de la surprise ${ }^{2}$. Certes, les occurrences du terme sont chez lui plutôt rares, mais, en concevant la réalité rendue à sa temporalité fondamentale comme une «création continue d'imprévisible nouveauté », selon un leitmotiv qui apparaît à partir de 1907 et de L'Évolution créatrice, c'est tout naturellement que la surprise s'impose chez lui comme le mode de donation privilégié

\footnotetext{
${ }^{1}$ C'est notamment le cas de P. Ricœur dans Philosophie de la volonté I. Le volontaire et l'involontaire, Paris, Aubier, 1950 (p. 238 et s.) ; H. Maldiney dans Penser l'homme et la folie, Grenoble, Jérôme Millon, 1991 ; Cl. Romano, dans L'Événement et le temps (III, 14, a: "L'attente et la surprise "), Paris, PUF, «Épiméthée », 1999 ; J.-L. Marion, dans Étant donné (§ 26), Paris, PUF, «Épiméthée ", 1997, et Reprise du donné (§ 33 : «La surprise), Paris, PUF, «Épiméthée », 2014 ; J. Benoist, dans Le Bruit du sensible (V : «Les surprises de la perception »), Paris, Le Cerf, 2013, mais déjà dans « La pensée et l'événement », in Archives de philosophie, n 59, 1996.

${ }^{2}$ Cette proximité ne doit cependant pas masquer les tensions et les différences entre Bergson et la phénoménologie, notamment et surtout avec Husserl, son contemporain. Sur ce point, voir F. Worms: "La conscience ou la vie ? Bergson entre phénoménologie et métaphysique », in Annales bergsoniennes II, Paris, PUF, « Épiméthée », 2004.
} 
d'un réel toujours en train de se faire. De fait, Bergson ne cesse d'opposer deux points de vue sur les choses, l'un, celui de l'intelligence, qui n'admet ni la nouveauté ni l'imprévisibilité, n'apercevant à travers l'espace, et pour les besoins de l'action, que des parties actuelles juxtaposées, l'autre, celui de l'intuition, selon lequel au contraire « la réalité est croissance globale et indivisée, invention graduelle, durée : tel, un ballon élastique qui se dilaterait peu à peu en prenant à tout instant des formes inattendues $»^{3}$. En sens inverse $d^{\prime}$ une conception qui circule d'Aristote à Heidegger en passant par Spinoza, Bergson permet alors de saisir la surprise non pas de manière négative, comme défaut ou déficit (de compréhension ou de connaissance), ni comme défaillance (de l'attention) ou paralysie (de l'action), mais comme expérience d'un surcroît de l'être. Que, dans la préparation d'un verre d'eau sucrée, pour reprendre le célèbre exemple de Bergson $^{4}$, je doive attendre que le sucre fonde, cela signifie que le réel, avant même de déjouer mon attente (ou de la remplir), est ce qui, plus fondamentalement, me fait attendre, révélant par là l'existence d'un rythme propre d'un réel qui, indépendant de moi en tant qu'il se déploie temporellement, n'est jamais le produit de ma volonté, et qui, précisément parce qu'il échappe à mon emprise, peut être source de surprise ${ }^{5}$. Mais toute l'originalité de la conception bergsonienne réside cependant dans la thèse selon laquelle le réel n'est pas potentiellement ni éventuellement, mais continuellement cause de surprise. Simultanément, c'est l'ensemble des coordonnées traditionnelles de la surprise qui se trouvent renversées, et comme inversées point par point, par Bergson, celle-ci apparaissant chez lui non pas comme discontinue, mais comme continue, non pas pétrifiante, mais dynamisante, non pas involontaire et adventice, mais conquise à la faveur d'un effort sur soi (que Bergson appelle « intuition »), non pas strictement corporelle, mais proprement métaphysique. Pour autant, Bergson n'ignore pas la structure typique de la surprise comme émotion paralysante, il lui accorde même une place et des analyses précises, mais distinctes, dans le cadre de ce qu'on pourrait appeler une anthropologie de l'événement ou de la situation extrême ${ }^{6}$, centrée sur une

\footnotetext{
${ }^{3}$ H. Bergson, La Pensée et le mouvant, Paris, PUF, «Quadrige », 2009, p. 105.

${ }^{4}$ H. Bergson, L'Évolution créatrice, Paris, PUF, «Quadrige», 2007, p. 9-10 ; p. 338-339.

${ }^{5}$ De ce point de vue, Bergson déplacerait le sens de la fameuse formule de Maldiney selon laquelle le réel est « ce qu'on ne peut pas attendre » (Penser l'homme et la folie, op. cit., p. 257). Si le réel nous surprend selon Bergson, ce n'est pas en raison de l'impossibilité, mais de la nécessité de l'attendre.

${ }^{6} \mathrm{~J}$ 'emprunte cette expression à Philippe Zawieja, spécialiste des risques psychosociaux, dont c'est l'un des objets d'étude d'un travail en cours. Appliquée à Bergson, elle désignerait assez bien l'analyse proposée dans le deuxième chapitre des Deux Sources de la morale et de la religion,
} 
nouveauté soudaine et destructrice, qui serait, sur le plan pragmatique et anthropologique, comme le pendant d'une nouveauté continue et créatrice sur le plan métaphysique. Il y aurait donc chez Bergson comme deux voies d'approche possibles de la surprise, entre lesquelles se distribueront les philosophies contemporaines qui la thématiseront, l'une centrée sur les dimensions du choc et de la crise, l'autre sur une temporalité féconde et créatrice ${ }^{7}$. Mais l'originalité profonde de Bergson résiderait peut-être au-delà de cette dualité, dans une sorte d'activation de la surprise, à la faveur d'un déplacement de l'être surpris (par la création continue de nouveauté ou par l'effraction soudaine d'une nouveauté destructrice), à l'acte de surprendre, au double sens de cette expression : introduire de la contingence et de l'imprévisible dans le monde et prendre quelque chose sur le fait, le saisir sur le vif, en train de se faire, selon une coïncidence entre "agir» et "voir», qui, dans toute l'œuvre de Bergson, définit aussi bien l'intuition métaphysique que l'acte libre. Il y aurait donc trois sens possibles de la surprise chez Bergson, selon qu'on l'envisage à travers la nouveauté, l'événement et l'acte libre.

\section{La nouveauté}

Le mot "surprise ", on l'a dit, n'est pas courant sous la plume de Bergson. On en trouve cependant une occurrence tardive et remarquable dans son œuvre, en 1934, dans les toutes premières pages de l'introduction à La Pensée et le mouvant. Dans ce texte-testament qu'il fait paraître sept ans avant sa mort, et dans lequel il retrace sur un registre autobiographique les origines de sa propre philosophie, Bergson évoque en effet "une surprise», dont il fait le point de

des réponses et des réactions de défense du psychisme dans certaines situations, périlleuses et soudaines (accident ou catastrophe). C'est dans ce cadre, il en sera question dans notre deuxième partie, que Bergson aborde la stupeur qui surgit dans les situations de grand danger.

7 Un tableau des philosophies contemporaines de la surprise serait l'objet d'un autre travail. Du côté de la surprise heureuse, on pourrait placer Bachelard dans sa philosophie de l'imagination poétique (sur ce point nous renvoyons à notre article à paraître : "Bachelard et Bergson" : un véritable chant en l'honneur du nouveau" »), ou J. Wahl (dans le prolongement de James et Whitehead); du côté de la surprise catastrophique, on peut mentionner C. Malabou (et la place qu'elle accorde au trauma, notamment dans L'Ontologie de l'accident. Essai sur la plasticité destructrice, Paris, Léo Scheer, 2009). Il faudrait sans doute compliquer et compléter cette dualité par deux approches intermédiaires, l'une neutre, en quelque sorte, qui ferait sobrement de la surprise une structure de la perception (voir par exemple J. Benoist, Le bruit du sensible, op. cit.), une autre encore qui maintiendrait son ambivalence, selon une dualité qui encadre littéralement l'existence, entre émerveillement et indignation (comme chez V. Jankélévitch), ou entre ressource créatrice et menace de destruction (voir par exemple F. Worms, Revivre, Paris, Flammarion, 2012). 
départ et le centre de toute son œuvre. Dans sa " première jeunesse ", entre 1881 et 1883, à sa sortie de l'École normale, son intention était, explique-t-il, de reprendre la philosophie de Spencer, non sans la rectifier sur certains points, et notamment en mécanique. C'est alors qu'un événement se produit, que Bergson relate dans ces termes :

Nous aurions voulu reprendre cette partie de son œuvre, la compléter et la consolider. Nous nous y essayâmes dans la mesure de nos forces. C'est ainsi que nous fûmes conduit devant l'idée de Temps. Là, une surprise nous attendait ${ }^{8}$.

Tel est donc cet événement, suggéré par les deux termes qui encadrent ce passage: le projet d'une reprise interrompu par une surprise. En effet, la confrontation de Bergson avec la notion de temps, qui devait n'être qu'un chapitre parmi d'autres d'un programme de consolidation théorique, est venue de manière inattendue déjouer le projet initial de prolonger Spencer, contraignant Bergson à commencer quand il n'aurait voulu que continuer. Car c'est bien sous le signe de la radicalité d'un commencement, au lieu d'un recommencement, que Bergson entend placer le point de départ de sa philosophie, rejouant par là le geste cartésien du début des Méditations, parfois même jusqu'à la parodie, comme il apparaît dans l'une des nombreuses variantes qu'il donne de ce récit, où il évoque la décision, prise dans le sillage de cette découverte inattendue, de rompre avec son passé et de « rejeter $[. .$.$] tout ce q u^{\prime}[i 1]$ avai[t] accepté jusqu'alors ${ }^{9}$.

Mais à travers l'invocation d'une " surprise », c'est immédiatement une distance avec Descartes qui se creuse, Bergson paraissant bien plutôt renouer avec la célèbre conception grecque de la philosophie comme étonnement, telle qu'on la trouve chez Aristote ${ }^{10}$ reprenant Platon ${ }^{11}$, et qui se trouverait donc ici réactivée et réhabilitée pardessus la critique cartésienne de l'admiration justement dirigée contre le thaumazein aristotélicien, dans lequel Descartes n'apercevait qu'un

\footnotetext{
${ }^{8}$ H. Bergson, La Pensée et le mouvant, op. cit., p. 2. Bergson tient manifestement beaucoup au mot «surprise » pour rendre compte de ce qu'il considère comme l'origine de sa philosophie. On le retrouve en effet dans une lettre à Giovanni Papini datée du 21 octobre 1903, (reprise dans le dossier critique de l'Essai sur les données immédiates de la conscience, Paris, PUF, 2010, (Essai dans la suite), p. 282), dans laquelle Bergson écrit: " $C^{\prime}$ est ainsi que je fus conduit à m'occuper de l'idée de temps. Je m'aperçus, non sans surprise, qu'il n'est jamais question de durée proprement dite en mécanique, ni même en physique, et que le "temps" dont on y parle est tout autre chose ".

${ }^{9}$ Lettre de Bergson à William James du 9 mai 1908, Essai, « Dossier critique », op. cit., p. 283.

${ }^{10}$ Voir Aristote, Métaphysique, A, 2.

11 Voir Platon, Théétète, 155 d.
} 
moment de stupeur initiale affectant l'esprit dans sa rencontre avec quelque objet nouveau, inconnu de lui par conséquent ${ }^{12}$.

Bergson serait pourtant très loin de se revendiquer d'Aristote, lui dont toute la philosophie veut être au contraire une réaction contre les Grecs, contre la manière grecque de penser, accusée d'avoir expulsé le temps au profit de l'éternité des essences. De fait, et sans que Bergson le précise lui-même directement ni explicitement, cette surprise inaugurale doit être envisagée tout autrement que de manière négative comme la marque d'une ignorance, ce qu'elle est bien chez Aristote ${ }^{13}$. L'origine de cette surprise est moins à rechercher du côté d'une limite de l'esprit, que dans l'épreuve d'une résistance, signe de son effectivité, du réel lui-même, son caractère surprenant attestant qu'il ne peut être ni construit, ni déduit, par la pensée, mais seulement rencontré par elle. Et Bergson ne cessera de répéter que c'est non seulement sans l'avoir voulu qu'il est venu buter sur la durée, mais que c'est même en contrariant sa nature qu'il a dû se détourner de la théorie des sciences pour se consacrer à la psychologie et à la métaphysique, seules à même selon lui de conduire à la compréhension rigoureuse du temps. S'en remettre à l'expérience de la surprise est par conséquent le signe d'une pensée qui ne décide pas elle-même intégralement des conditions de son exercice, mais qui accepte l'épreuve des faits en se laissant contraindre et instruire par eux. C'est ainsi qu'il faut comprendre la revendication bergsonienne d'un "empirisme vrai », qu'il oppose à toute tendance logique ou systématique de la pensée, dont la caractéristique est de sortir du temps et de l'expérience réelle, pour se donner elle-même son objet. Cette exigence d'empirisme est même la seule cohérente pour une pensée qui place le temps au cœur de sa démarche. Car Bergson ne se contente pas de découvrir dans la succession temporelle un principe de production en vertu duquel le réel se crée continuellement de manière imprévisible sans jamais se donner en une fois et complètement, mais il fait de cette découverte le principe de sa propre progression philosophique, déclarant à plusieurs reprises qu'aucun des résultats de ses livres n'auraient pu être déduit du livre antérieur, manière de dire que chacun est une surprise, pour son lecteur, mais d'abord pour son auteur. Plus encore, on s'aperçoit en le lisant que la notion de durée elle-même, que

\footnotetext{
12 Sur ce point, voir notamment, Descartes, Traité des passions, art. 73, AT XI, 382-383. Sur la critique cartésienne de la conception aristotélicienne de l'étonnement, voir L. Renault, Descartes ou la félicité volontaire, Paris, PUF, « Épiméthée », 2000, p. 20 et sq.

${ }^{13}$ Voir Aristote, Métaphysique, A, 2, 982 b 17 : «Apercevoir une difficulté et s'étonner, c'est reconnaître sa propre ignorance ». Cette reconnaissance conduit aussitôt à l'effort pour sortir de l'ignorance.
} 
Bergson invoque constamment comme une sorte d'idée fixe, n'est pas principe ontologique de changement sans changer elle-même, puisque, d'un livre à l'autre, elle prend des aspects à chaque fois différents, à chaque fois nouveaux ${ }^{14}$.

Pourtant, ce n'est pas le temps lui-même qui d'emblée surprend Bergson. Sa surprise porte plutôt sur l'étrange et inattendue incapacité des sciences, spécialement des mathématiques, à atteindre le temps dans sa réalité la plus simple, la plus immédiate, la plus sensible, qu'il appelle "durée ». Bergson écrit ainsi : "Nous fûmes très frappé de voir comment le temps réel [...] échappe aux mathématiques ${ }^{15}$; ou encore, dans un autre passage: "Je m'aperçus à mon grand étonnement que le temps scientifique ne dure pas $\gg^{16}$. La surprise de Bergson porte sur la découverte d'une chose étrange, du même ordre que ces objets impossibles, impossibilia, que sont un cercle carré ou une montagne sans vallée: un temps qui ne dure pas. Comment est-ce possible ? C'est ce qui est élucidé par Bergson à travers une genèse critique des opérations de mesure et de calcul auxquelles les sciences soumettent le temps en lui imposant une nécessaire dénaturation. Car pour mesurer le temps, il faut en effet 1/ l'immobiliser, lui qui est par principe mobilité et changement, en lui assignant des «moments » ou des « parties »; 2/ ne considérer que la forme du passage d'un instant à un autre, valable pour n'importe quel contenu, le temps n'étant plus alors qu'un cadre général, neutre et indifférent, une série indéfinie d'instants quelconques et de simultanéités. Immobilité, partie, cadre: le temps se voit ainsi attribué, pour les besoins de sa mesure, des caractéristiques qui appartiennent à l'espace. Mais précisément ce sont les conditions de la surprise qui disparaissent avec l'espace, dès lors qu'il n'y a plus succession de moments, mais simple coexistence de points ou de simultanéités. Or, « entre les simultanéités se passera tout ce qu'on voudra, le temps pourrait s'accélérer énormément, et même infiniment, rien ne serait changé pour le mathématicien, pour le physicien, pour l'astronome ${ }^{17}$. Par le calcul, l'éclipse de lune qui se produira dans dix ans,

\footnotetext{
${ }^{14}$ Dans un entretien tardif, daté de 1936, avec Jean de la Harpe, Bergson confiait à son interlocuteur ceci qui prend toute sa portée à la lumière de la surprise : " Malheureusement, voyezvous, mes livres ne sont pas toujours cohérents entre eux : le temps de L'Évolution créatrice ne "colle" pas avec celui des Données immédiates ». C'est ainsi notamment que le centre de gravité de l'analyse du temps ne cesse de se déplacer au fil de l'œuvre, l'Essai insistant surtout sur le " présent » de l'acte libre, Matière et mémoire sur le " passé ", L'Évolution créatrice sur l'« avenir ». ${ }^{15}$ H. Bergson, La Pensée et le mouvant, Paris, PUF, « Quadrige », 2009, p. 2.

${ }^{16}$ Lettre de Bergson à William, in Essai, «Dossier critique», op. cit., p. 283. C'est Bergson qui souligne.

17 H. Bergson, La Pensée et le mouvant, op. cit., p. 3.
} 
a en quelque sorte déjà eu lieu, car sa position dans l'espace à cette date est déjà donnée à l'astronome. Surtout, ces dix années n'ont pour lui dans le calcul aucune réalité, tandis que du point de vue de sa durée individuelle, une seule seconde contribuera à colorer différemment et à transformer totalement toute sa personne.

Pour autant, cette genèse critique du temps mesuré ne suffit pas à dissiper cette surprise devant un temps qui ne dure pas. C'est que nous ne sommes pas là devant une erreur rectifiable une fois pour toutes, mais plutôt face à une illusion générale et constitutive de l'esprit humain, à une tendance fondamentale inscrite en lui, qui, même dénoncée, ne cesse de renaître. La surprise qu'évoque Bergson ne désigne donc pas seulement la ponctualité d'une prise de conscience individuelle, un simple épisode autobiographique, mais la surprise partageable et répétable de toute conscience pure du temps confrontée à son appréhension spatiale, scientifique ou vulgaire. Il ne $\mathrm{s}^{\prime}$ agit donc pas pour Bergson de raconter une surprise, sans en même temps s'efforcer de la susciter, de la faire naître en chacun de nous.

Or, à partir de 1907 et de L'Évolution créatrice, la surprise semble gagner un véritable fondement, à travers l'apparition d'un aspect de la durée qui passe désormais au premier plan, sa nouveauté : "Plus nous approfondirons la nature du temps, plus nous comprendrons que durée signifie invention, création de formes, élaboration continue de l'absolument nouveau ${ }^{18}$. La nouveauté qui, dans le domaine de la biologie, prend la forme sensible de l'évolution des espèces, oblige Bergson à concevoir la vie dans son ensemble sur le modèle analogique d'une conscience qui prolonge les moments d'une continuité temporelle les uns dans les autres, sans quoi il n'y aurait qu'une pure et simple répétition du même.

Mais Bergson ajoute aussitôt un critère capital à celui de la nouveauté, celui de l'imprévisibilité, qui ne renvoie à aucune dissolution de l'expérience, à aucun déficit de rationalité, mais au contraire à un excès du réel sur la pensée qui ne saurait en anticiper les formes à venir $^{19}$. Cela signifie que chaque moment du temps n'est pas seulement nouveau pour une conscience, mais imprévisible en soi et en droit. Si la nouveauté et l'imprévisibilité sont les deux critères désormais inséparables de la durée et de sa nature profonde, alors la saisie du temps réel ne peut être que de l'ordre de la surprise. Pourtant, cette surprise paraît moins surgir directement du rapport à

${ }^{18}$ H. Bergson, L'Évolution créatrice, Paris, PUF, «Quadrige », 2007, p. 11.

${ }^{19}$ Voir sur ce point les analyses de F. Worms, dans Bergson ou les deux sens de la vie, Paris, PUF, «Quadrige », p. 201. 
la durée, que d'un écart entre la conscience réfléchie et spatiale du temps, et sa conscience immédiate et pure, et dans un moment critique où il s'agit pour Bergson de confronter la tendance spatialisante de notre esprit (considérer le réel comme s'il n'y avait pas de temps) à son incapacité de saisir la réalité du temps. Il lance comme un défi à chacun : "Essayez de vous représenter aujourd'hui l'action que vous accomplirez demain $»^{20}$. Si je peux bien «imaginer » dès maintenant le «mouvement à exécuter», ce que je penserai le moment venu en l'exécutant ne pourra surgir qu'à la faveur d'une surprise, puisque le temps écoulé lui ajoutera quelque chose que je suis incapable de rejoindre à présent par la seule pensée.

Cet écart est précisément le point de départ de Bergson dans son article de 1930 sur « Le possible et le réel ». Bergson commence par constater l'incapacité de chacun de produire le réel à volonté. En accumulant un nombre aussi grand que je veuille de détails ou d'éléments, jamais je n'obtiendrai dans sa simplicité, et avant qu'il ne $\mathrm{m}^{\prime}$ arrive, le moindre événement, l'événement le plus banal qui soit : "J'ai beau me représenter le détail de ce qui va m'arriver : combien ma représentation est pauvre, abstraite, schématique, en comparaison de l'événement qui se produit! ${ }^{21}$. L'exclamation est ici comme la marque de la surprise devant l'abîme impossible à combler entre la représentation anticipée de l'événement et sa survenue effective. Pourquoi ? Qu'est-ce qui n'est pas donné ni donnable à la pensée toute seule? Qu'est-ce qui définitivement et constitutivement échappe à son pouvoir d'anticipation? Pour le dire, Bergson s'appuie sur un exemple à première vue déroutant. Non pas celui $d^{\prime}$ 'un grand événement, spectaculaire, unique, retentissant, dont chacun reconnaîtrait immédiatement le caractère inattendu, mais celui, volontairement banal, trivial, d'une réunion à laquelle je dois assister, événement qui en est à peine un, à la fois fréquent, programmé, aisément anticipable, le moins surprenant qui soit. Si néanmoins la réunion constituera bien un événement pour moi, c'est dans la mesure où sa part d'inattendu ne tient pas tant à ce qui m'arrivera, qu'à la manière ou à la façon dont cela m'arrivera. Or, cette manière tient à un «imprévisible rien qui change tout ${ }^{22}$. Voilà ce qui ne peut être anticipé, ce qui excède la pensée, et sans doute toute description possible, et qui nous conduit donc au-delà de toute phénoménologie : ce qui ne peut être ni prédit, ni décrit, et qui n'est pas à strictement

\footnotetext{
${ }^{20} \mathrm{H}$. Bergson, La Pensée et le mouvant, op. cit., p. 10.

${ }^{21} \mathrm{H}$. Bergson, «Le possible et le réel », in La Pensée et le mouvant, op. cit., p. 99.

22 Idem.
} 
parler un vécu, mais se confond avec la manière dont la vie est vécue. Il revient sans aucun doute à Vladimir Jankélévitch d'avoir poussé le plus loin l'effort pour approcher ce « rien », dans le sillage de Bergson justement, dans Le Je-ne-sais-quoi et le Presque-rien ${ }^{23}$. Ce qui toujours nous surprendra, ce qui à chaque fois nous prend par surprise, c'est cet indescriptible qui est, selon Jankélévitch, la marque infinitésimale du réel, "l'odeur d'effectivité » qui distingue une fois pour toutes l'événement réel de sa simple possibilité, l'«invisible supplément », le «milliardième de milligramme en plus du concept ${ }^{24}$ qui donne à quelque chose d'exister au lieu d'être simplement pensé, un ineffable qui distingue l'hypothétique de l'effectif. La surprise est ce qui signe de manière irrécusable l'entrée dans le réel.

Or, le propre de ce « rien » est de constituer la trame, et comme le grain ou la texture, de toute notre expérience, de toute expérience, et non seulement de certaines expériences exceptionnelles. Bergson distingue en effet d'un côté ce qui, de la réunion, peut être connu à l'avance: «je sais quelles personnes j'y trouverai, autour de quelle table, dans quel ordre, pour la discussion de quel problème $»^{25}$, et de l'autre, ce qui ne peut être anticipé, la réunion en tant qu'événement : «qu'elles viennent, s'assoient et causent comme je m'y attendais, qu'elles disent ce que je pensais bien qu'elles diraient : l'ensemble me donne une impression unique et neuve $»^{26}$. La part de surprise de la réunion ne réside pas dans ce qui y dit, pas davantage surgira-telle d'un imprévu, d'une circonstance, par exemple à la condition qu'elle n'ait pas lieu. Cette surprise tient au fait même qu'elle ait lieu. Telle est ici l'audace de Bergson : même ce qui a lieu conformément à mon attente (« ...qu'elles viennent, s'assoient et causent comme je $m^{\prime} y$ attendais », etc.), me surprend, par sa manière d'avoir lieu en m'arrivant. Par là, Bergson propose de concevoir la surprise de manière originale, tout autrement que comme une attente non remplie ou déjouée. Même lorsque mon attente est remplie ou confirmée, le réel m'affecte d'une manière qui reste absolument originale et inanticipable.

C'est alors que Bergson envisage l'objection suivante : il n'est pas surprenant que ce qui m'arrive me surprenne, dans la mesure où je ne choisis pas ce qui m'arrive, et qu'il y a nécessairement des circonstances indépendantes de moi, et que c'est même la définition

\footnotetext{
${ }^{23}$ Voir V. Jankélévitch, Le Je-ne-sais-quoi et le Presque-rien, Paris, Seuil, 1980, p. 104 et sq.

${ }^{24}$ Idem.

${ }^{25}$ H. Bergson, La Pensée et le mouvant, op. cit., p. 99.

${ }^{26}$ Ibid., p. 100.
} 
et le critère de la perception et de l'expérience, échapper à ma volonté $^{27}$. Or, pour Bergson, ce n'est pas seulement l'extériorité perceptive qui est source de surprise, mais notre propre intériorité, y compris dans sa dimension la plus contrôlée, la moins aléatoire, l'acte volontaire et libre, dont le propre est qu'il « réalise du voulu et par conséquent du prévu ${ }^{28}$. Bergson propose d'envisager la surprise à la faveur d'un déplacement remarquable: non pas à travers l'activité intentionnelle de la conscience, mais à travers la conscience d'un acte intentionnel. Or, l'impression de nouveauté n'est pas présente, à part égale dans la conscience du moi agissant et dans la perception du monde, mais bien "plus vive que jamais »! Bergson va même encore plus loin : ce qui de notre propre acte nous surprend, ce ne sont pas ses effets ou ses conséquences éventuels ${ }^{29}$, mais sa face subjective, les " moments de la délibération », qui ont beau dépendre de moi, n'en sont pas moins chacun surprenant, ce qui doit conduire à envisager le rapport à soi selon une extériorité analogue à celle que nous entretenons avec les choses, mes états intérieurs se produisant à mes propres yeux comme un spectacle: «j'ai la même impression de nouveauté devant le déroulement de ma vie intérieure ${ }^{30}$. Il y a ainsi une succession temporelle de mes états qui est inéliminable, en vertu de laquelle j'assiste à ma vie intérieure comme j'assiste à une réunion, ce qui explique que je puis éprouver de la surprise en les considérant, d'une manière analogue à ce que l'artiste éprouve devant sa propre création.

Parvenu à ce point, une difficulté se profile cependant : celle d'envisager une surprise continue qui serait comme la doublure et le corrélat d'une nouveauté continue. De fait Bergson ne décrit pas l'expérience de la nouveauté en terme de surprise, même si tout semble appeler un tel terme dans ses analyses. Lorsque, dans L'Évolution créatrice, il oppose l'attitude spontanée de l'intelligence qui s'étonne de trouver dans le monde tant d'ordre dans une complexité si grande, à celle de l'esprit intuitif devant la nouveauté, Bergson introduit les termes «admiration » et " étonnement»: "Mais ce qui est admirable en soi, ce qui mériterait de provoquer l'étonnement, c'est la création sans cesse renouvelée que le tout du réel accomplit en

\footnotetext{
27 Voir J. Benoist, Le bruit du sensible, op. cit., p. 140.

${ }^{28} \mathrm{H}$. Bergson, La Pensée et le mouvant, op. cit., p. 100.

${ }^{29}$ On peut penser à la thématique hégélienne des circonstances de l'action dans le paragraphe 132 des Principes de la philosophie du droit, qui mériterait peut-être d'être relu à la lumière de la question de la surprise dans l'action, en tant que ses effets peuvent nous échapper.

${ }^{30} \mathrm{H}$. Bergson, La Pensée et le mouvant, op. cit., p. 100.
} 
avançant ${ }^{31}$. Si Bergson recourt ici au conditionnel, c'est pour suggérer qu'un étonnement intuitif devant la création continue de la réalité ne se produit pas de lui-même, mais devrait être conquis. On pourrait toutefois proposer une autre lecture de ce conditionnel et soutenir que, à la limite, l'intuition ne connaîtrait pas la surprise, dans la mesure où elle est simplement accueil de ce qui se déploie temporellement. L'étonnement spontané de l'intelligence devant le réel, comme Bergson le montre dans le premier chapitre de L'Évolution créatrice, consiste à s'émerveiller de la complexité de structure des choses observées dans la nature, et singulièrement dans le domaine du vivant, comme c'est le cas devant un organe tel que l'œil, dont l'arrangement des parties paraît si extraordinaire, que l'intelligence ne peut s'empêcher d'y apercevoir le plus "formidable des travaux $\mathrm{d}^{\prime}$ Hercule » accompli par la nature ${ }^{32}$. Or, Bergson montre que cette complexité n'est qu'un effet de perspective, qui ne se produit qu'à partir d'un point de vue extérieur pris sur un processus intérieurement simple, sur le modèle du bras qu'on lève, qui semble parcourir, à celui qui l'observe, une série de positions successives dans l'espace, et qui n'est en réalité qu'un mouvement simple et indivisible pour celui qui accomplit le geste. De même, le processus par lequel la vie produit un œil, est lui aussi une chose simple, et, de la simplicité, il n’y a pas lieu de s'étonner : "La nature, écrit Bergson, n'a pas eu plus de peine à faire un œil que je n'en ai à lever la main $\gg^{33}$.

Mais peut-être que du point de vue de l'espace, inversement, il n'y aurait pas davantage place pour la surprise, ainsi qu'on le remarquait plus haut. Dans l'espace en effet, tout est donné, tout se juxtapose, et "aucune complication de l'ordre mathématique avec lui-même, si savante qu'on la suppose, n'introduira un atome de nouveauté dans le monde ${ }^{34}$. Pour cela, il faudrait se replacer dans la succession temporelle.

Il semblerait donc que sur le plan intellectuel, du point de vue de l'espace pur, aussi bien qu'à l'autre extrémité, sur un plan purement intuitif et temporel, il n'y ait pas de surprise. Ce n'est peut-être finalement qu'entre ces deux plans, ou plutôt à leur jonction, que s'ouvre la possibilité de la surprise, quand l'intelligence se trouve confrontée aux effets inéliminables de la durée, et qu'elle éprouve une résistance du réel, qui souvent apparaît sous la plume de Bergson

\footnotetext{
${ }^{31} \mathrm{H}$. Bergson, L'Évolution créatrice, op. cit., p. 218.

32 Ibid., p. 92.

${ }^{33}$ Idem.

${ }^{34}$ Ibid., p. 218.
} 
à travers l'expression «J'ai beau faire... »: j'ai beau me représenter un événement à l'avance, je suis incapable de construire du réel...

Pourtant Bergson n'en reste pas là. La surprise n'est pas seulement le résultat de ce choc entre la conscience réfléchie et la conscience immédiate du temps. Car si la nouveauté métaphysique, continue et créatrice, est l'objet d'une conquête, d'un effort philosophique, qui est toute l'affaire de Bergson dans son œuvre, il est une nouveauté qui s'impose dans une expérience, celle, paralysante, de l'événement catastrophique et destructeur, de l'accident ou du drame, dont la structure est au cœur du deuxième chapitre des Deux Sources de la morale et de la religion.

\section{L'événement}

Cette analyse critique et métaphysique n'épuise donc pas le sens de la surprise dans la philosophie de Bergson. Dans le deuxième chapitre de son dernier livre, Les Deux Sources de la morale et de la religion, celui-ci consacre un important passage à la surprise ${ }^{35}$, selon une tout autre perspective, anthropologique cette fois, où il s'agit de décrire un certain nombre de mécanismes d'ajustement typiques mis en œuvre par l'homme dans les situations de rupture de ses liens pragmatiques avec le monde. Pour saisir de manière plus précise les attendus de cette nouvelle perspective, il convient peut-être de commencer par la situer par rapport aux enjeux du chapitre dans lequel elle se situe, ceux d'une théorie de la religion et d'une analyse de l'origine des croyances religieuses.

À la manière d'un Hume, Bergson propose dans ce chapitre une genèse naturelle de la religion. Le comportement religieux y est envisagé comme un fait universel de la nature humaine, et la religion comme un ensemble de réactions que la nature a placées en l'homme en guise de réponses pratiques à certains problèmes vitaux fondamentaux: prise de conscience de la mort, incertitude du futur, aléas de l'existence, etc. Or, la surprise est à envisager comme un tel problème dans les situations exceptionnelles où l'homme ne peut plus agir ni réagir, lorsque sa prise ou son emprise sur les choses n'est plus possible, comme c'est exemplairement le cas dans l'expérience d'une grande catastrophe. La surprise apparaît alors comme la «crainte qui paralyse», issue de «la pensée que des forces

\footnotetext{
35 Voir H. Bergson, Les Deux Sources de la morale et de la religion, Paris, PUF, «Épiméthée », 2008, p. 159-167.
} 
formidables et aveugles sont prêtes à nous broyer inconsciemment $»^{36}$. À bien des égards, la situation qui intéresse ici Bergson est celle qui est précisément écartée par Kant dans la Critique de la faculté de juger, lorsqu'il fait de la sécurité du spectateur face au déchaînement des forces de la nature, la condition indispensable pour que surgisse le sentiment du sublime ${ }^{37}$. L'objet de Bergson est bien, au contraire, celui de la crainte ou de la frayeur, et la question qu'il pose est la suivante: que se passe-t-il lorsque brusquement nous nous sentons en danger? Non pas face à un danger minime, mais devant un grand danger? Qu'arrive-t-il quand l'homme qui est fait pour agir, ne peut plus agir?

$C^{\prime}$ est alors que surgit la surprise, que Bergson envisage comme une conduite vitale, à un stade où l'émotion devient paralysante, et par conséquent préjudiciable à l'individu qui ne peut plus réagir. La surprise n'est plus ici, comme c'est le cas sur le plan métaphysique, relative à une continuité, mais elle est corrélative d'une rupture. En ôtant tout pouvoir d'initiative, cette stupeur constitue un obstacle au souci de conservation, ce qu'elle n'est pas à l'origine puisqu'elle est aussi bien, comme le fait remarquer Bergson (reprenant James reprenant Darwin), ce qui dispose à la fuite. Mais au-delà d'un certain seuil, elle immobilise, selon la caractéristique primitive de l'admiration-surprise soulignée par Descartes dans Les Passions de l'âme $e^{38}$, et que Bergson paraît ici retrouver, non sans la faire refluer du jugement de connaissance vers l'action. Mais ce n'est pas ici un " objet», rare ou extraordinaire qui suscite la surprise, mais une situation exceptionnelle, lorsque surgit un péril "grave et soudain », comme c'est le cas dans les grands bouleversements du type catastrophe naturelle qu'évoque Bergson, «tremblement de terre, inondation, ouragan », et qu'il envisage à travers le témoignage de William James présent en Californie lors du séisme de San Francisco en avril 1906, ou encore dans celui de l'accident (Bergson évoque cette fois un souvenir d'enfance, celui d'une chute de cheval), ou enfin d'un drame historique (Bergson évoque la déclaration de guerre de l'Allemagne à la France, le 4 août 1914). Dans ces pages, la surprise cependant n'apparaît pas seulement, ni d'abord et de manière étroite, comme un affect, mais elle désigne à la fois un ensemble de

\footnotetext{
${ }^{36}$ Ibid., p. 164.

${ }_{37}$ Voir I. Kant, Critique de la faculté de juger, § 28, trad. A. Renaut, Paris, Flammarion, 1995, p. 243-245 : «Mais, plus leur spectacle est effrayant, plus il ne fait qu'attirer davantage, pourvu que nous nous trouvions en sécurité ».

${ }^{38}$ R. Descartes, Les Passions de l'âme, II, 73: "Tout le corps demeure immobile comme une statue ».
} 
circonstances extrêmes et la réaction d'un individu à cette situation tendanciellement paralysante. Elle n'est plus, comme sur le plan métaphysique, le corrélat de l'expérience considérée dans sa continuité temporelle, mais celui d'une expérience, ponctuelle, isolable, datable, historiquement et/ou biographiquement.

Mais dans l'analyse que Bergson propose de la surprise paralysante quelque chose de remarquable se produit. Dans les situations extrêmes où elle survient, tout se passe comme si elle était aussitôt empêchée, aussitôt neutralisée, pour permettre à celui qui s'y trouve plongé d'affronter la situation, au lieu de se laisser terrasser.

Une réaction défensive naturelle se déclenche en effet qui empêche la sidération devant le "péril grave et soudain». Cette réaction consiste à personnifier l'événement, à lui conférer quelque chose comme une unité et une individualité, qui en font une sorte d'être familier à qui on attribue des projets, des intentions, des idées, de telle sorte qu'une relation à lui s'établit, de l'ordre de la "camaraderie », comme pour répliquer à l'effet de défamiliarisation provoqué par l'événement :

Le tremblement de terre de San Francisco fut une grande catastrophe. Mais à James, placé brusquement en face du danger, il apparaît avec je ne sais quel air bonhomme, qui permet de le traiter avec familiarité. "Tiens, tiens! c'est ce vieux tremblement de terre ». Analogue avait été l'impression des autres assistants. Le tremblement était "pervers»; il avait son idée, «il s'était mis en tête de détruire ». On parle ainsi d'un mauvais garnement, avec lequel on n'a pas nécessairement rompu toute relation ${ }^{39}$.

Le processus décrit par Bergson est d'une certaine manière, pour emprunter et détourner un terme de Max Weber, celui d'une "quotidianisation » de l'événement, selon un mouvement d'intégration de l'événement dans le monde et dans le champ d'expérience de celui qu'il menace, par désactivation de sa charge dévastatrice et déstabilisatrice. Ce processus repose sur la production d'une «image qui rassure ", dont la fonction est de maintenir en alerte notre faculté d'agir et de réagir, qui autrement serait suspendue. En sens inverse de ce qui se passe sur le plan métaphysique, où le réel dépassait ou débordait la représentation anticipée de ce qui va arriver, l'image est ici ce qui vient envelopper l'événement, l'accueillir, l'amortir, en vue de nous redonner sur lui sinon une emprise, tout au moins une prise possible, dans l'instant même où tout lien sensori-moteur menaçait

${ }^{39}$ H. Bergson, Les Deux Sources de la morale et de la religion, op. cit., p. 163-164. 
d'être anéanti, ce afin de rétablir notre rapport au monde, grâce à une réaction de l'imagination travaillant au bénéfice de l'action, selon une fonction que Bergson appelle «fabulatrice», véritable machine à fabriquer des entités imaginaires, personnalités vagues ou élémentaires, avec lesquelles une relation est possible. Tout se passe comme si une fonction individuante ripostait immédiatement, naturellement, à la charge désindividuante du traumatisme que constitue l'événement catastrophique ${ }^{40}$. Cette réponse psychique, qui est selon Bergson à l'origine de la production de divinités chez les hommes, est toutefois distincte de celle qui consiste à attribuer un auteur (dieux ou démon) aux événements, puisqu'elle conduit à considérer l'événement lui-même comme un « agent vivant».

Mais, dans le même temps où la surprise se trouve neutralisée dans ce qu'elle aurait de paralysant, un affect apparaît, celui d'admiration, éprouvé devant le surgissement de l'événement dans le monde. Ce point est particulièrement important, il revient dans l'analyse des trois situations évoquées par Bergson, de manière d'abord discrète, dans le passage consacré à James, puis de plus en plus explicite et précis dans les deux cas suivants. La thèse qui se précise progressivement est originale: dans chaque cas, la stupeur devant l'inattendu se trouve relayée spontanément par une attitude de glorification de l'événement qui, de simple éventualité, est devenu réalité, dans une sorte de passage à l'être et à l'acte, et à la faveur $\mathrm{d}$ 'une véritable campagne victorieuse contre les obstacles à sa propre existence.

Bergson s'appuie d'abord sur James, qui, dans son expérience du séisme de San Francisco, évoque, dans un passage autobiographique longuement cité, une certaine émotion de l'ordre d'une " allégresse » ressentie "devant l'intensité de vie qu'une idée abstraite, une pure combinaison verbale comme "tremblement de terre" pouvait prendre, une fois traduite en réalité sensible et devenue l'objet d'une vérification concrète $»^{41}$. Contrairement à ce qu'on pourrait penser, l'événement ne surgit pas à partir de rien, d'un vide dans la représentation, mais de certaines attentes ou anticipations qui font qu'il était envisageable, voire envisagé, tout au moins de manière abstraite, comme une simple probabilité.

C'est sur ce même point que Bergson insiste le plus dans son dernier exemple, celui de sa découverte, en ouvrant le journal le

\footnotetext{
${ }^{40}$ Sur cette puissante désindividuante de l'événement traumatique, voir $\mathrm{Cl}$. Romano, L'Événement et le temps, op. cit., p. 261 et $s q$.

${ }^{41}$ H. Bergson, Les Deux Sources de la morale et de la religion, op. cit., p. 161.
} 
matin du 4 août 1914, de la déclaration de guerre de l'Allemagne à la France. À nouveau, Bergson insiste sur la surprise devant un événement pourtant prévisible. Bien que présente en effet dans tous les esprits après 1871, une guerre apparaissait, explique Bergson, " tout à la fois comme probable et comme impossible: idée complexe et contradictoire ». Elle conserva son caractère abstrait jusqu'aux heures tragiques où le conflit apparut comme inévitable, jusqu'au dernier moment, alors qu'on espérait contre tout espoir $»^{42}$. Comme tout à $l^{\prime}$ heure et sur un plan métaphysique, l'analyse de la surprise met en jeu les catégories du possible et du réel, de l'abstrait et du concret. Mais c'est une autre logique qui préside à leur rapport. Ici, la surprise ne naît plus tant de la différence ou de l'écart entre ce qui est pensé, ou conçu, d'un côté, et ce qui est réel de l'autre, mais bien plutôt du passage de l'abstrait au concret. Ce qui surprend, curieusement, ce n'est pas que l'attente soit déjouée, mais qu'elle soit remplie, que l'impossible si redouté se soit invité dans la réalité, installé, comme si le surgissement de l'événement venait tout à coup, par un effet de cristallisation, relier entre eux tous les petits signes du passé dont on comprend, par l'effet d'une révélation rétrospective, qu'ils étaient annonciateurs de sa venue.

Mais si la surprise est bien provoquée par l'entrée dans le réel, c'est non seulement par la factualité de la réalisation, par le fait que cela ait lieu ou soit arrivé, mais par la manière dont cela arrive : facilement. Bergson explique :

J'éprouvais ce que dit James, un sentiment d'admiration pour la facilité avec laquelle s'était effectué le passage de l'abstrait au concret : qui aurait cru qu'une éventualité aussi formidable pût faire son entrée dans le réel avec aussi peu d'embarras ? Cette impression de facilité dominait tout ${ }^{43}$.

L'admiration pour la facilité est ici remarquable. Elle paraît littéralement inverser l'étonnement intellectuel évoqué plus haut : ce qui surprend ici Bergson, ce n'est pas la difficulté rencontrée par l'événement pour s'imposer, les «travaux d'Hercule » dont il aurait eu à s'acquitter, mais bien au contraire la facilité, la désinvolture déconcertante avec laquelle l'événement fait irruption, s'installe dans sa vie, sans gêne, pourrait-on dire, à tous les sens de cette expression, à la fois au sens où il ne rencontre aucun obstacle, et où, envahissant et familier, il impose sa présence :

42 Ibid., p. 166.

43 Ibid., p. 167. 
Bergson et les sens de la surprise : nouveauté, événement, liberté

Il avait attendu son heure ; et sans façon, familièrement, il s'asseyait à sa place. C'est pour intervenir à ce moment, en cet endroit, qu'il s'était obscurément mêlé à toute mon histoire $[\ldots]^{44}$.

C'est l'événement qui a ici l'initiative, à la manière d'un personnage ou d'une personne, conformément aux effets de la fonction fabulatrice. C'est lui qui, au sens fort et actif du terme, surprend Bergson, mais aussi bien ses contemporains, tous réduits à la posture passive de simples spectateurs.

On mesure alors à quel point il n'est pas question ici de retrouver par la voie de l'anthropologie, la part métaphysique de nouveauté que contient le réel, celle qui est décrite et saluée au début de l'article sur «Le possible et le réel ». Car ce qui surprend n'est pas tant le partage entre l'image et le réel, entre la représentation anticipée et la production effective de l'événement, que le passage du possible au réel. Encore n'y a-t-il pas ici réellement surprise, car l'expérience de l'événement nous place devant l'apparition brutale de quelque chose dont la nouveauté, une fois traitée par la fonction fabulatrice, se résorbe bientôt dans la continuité d'une relation familière.

Parvenu à ce stade de l'analyse, un véritable chassé-croisé paraît alors s'établir entre les perspectives anthropologique et métaphysique de l'événement : si selon la première, la nouveauté catastrophique et visible, et même spectaculaire, est finalement toujours déjà neutralisée par l'imagination qui la banalise en la rabattant sur une configuration rassurante, selon la seconde, à l'inverse, il s'agirait, grâce à la philosophie, de retrouver et de rendre visible une création de nouveauté à l'inverse d'abord imperceptible (à notre intelligence), alors même qu'elle est continue.

Il reste que ces deux premières approches de la nouveauté constituent potentiellement deux voies d'extinction pour l'analyse de la surprise. Car du point de vue anthropologique, c'est aussi bien sa neutralisation et son dépassement qui sont visés par Bergson, tandis que sur le plan métaphysique, ce dernier ne décrit jamais directement l'expérience de la nouveauté comme une surprise, mais comme une « joie ». Or, ce dernier terme, bien loin de nous en éloigner, pourrait bien relancer la compréhension bergsonienne de la surprise dans une troisième direction. Car la joie implique d'une certaine manière la surprise. Contrairement au plaisir qui regarde toujours en arrière en visant la restauration d'un équilibre rompu par le besoin, et par là, la conservation de la vie, la joie est toujours pour Bergson le sentiment

44 Idem. 
qui accompagne l'ouverture d'un avenir à travers la création de quelque chose de nouveau ${ }^{45}$. L'artiste avec son œuvre, le savant à travers sa découverte, l'inventeur, la mère et son enfant, selon les exemples que donne Bergson, chacun éprouve la joie de se sentir créateur, c'està-dire d'avoir «appelé quelque chose à la vie » ${ }^{46}$, à la durée. Mais la surprise dont il s'agit n'est, on le voit, en aucun cas purement passive; elle est inséparable d'un acte, d'une conquête, d'une réussite, et c'est à la condition d'une initiative, et à la pointe de cet effort, que l'on pourra surprendre le réel, à la fois au sens où l'on fait surgir en lui quelque chose de nouveau, et où, par là, on découvre son principe, sa "destination», qui autrement nous resteraient masqués ou inaccessibles : la création. Du coup c'est à un retournement de la situation décrite par l'anthropologie de l'événement que nous sommes conduits, puisque ce n'est pas nous qui sommes surpris, mais nous qui surprenons. Loin que la surprise se laisse analyser selon la dynamique centripète d'une nouveauté adventice qui nous impose la passivité d'un choc ou d'un saisissement par lesquels nous sommes frappés d'arrêt, elle devrait être envisagée à partir de l'initiative d'un sujet, ce que Bergson soutient en réalité depuis le début, dès son premier livre, l'Essai, lorsqu'il fait de l'acte libre l'expérience privilégiée de la durée.

\section{L'acte libre}

Loin de se limiter au phénomène passif et négatif d'un ébranlement de l'action, la surprise prend dans la philosophie de Bergson une forme ainsi qu'une portée nouvelles, dès lors qu'on l'envisage et qu'on la reconsidère à la lumière et à partir non plus de l'événement (ce qui nous arrive), mais de l'action, et plus précisément de l'acte libre (ce que nous faisons arriver). Conçue du point de vue de l'acte libre en effet, la surprise change de sens et pour ainsi dire de signe, elle cesse de renvoyer à une passivité, et doit être considérée à la forme active: surprendre. Cet aspect n'est pas sans rapport avec le sens métaphysique de la surprise devant la nouveauté abordé dans notre premier moment. Mais ce qu'il faut dire et comprendre à présent, c'est que la sensibilité à la nouveauté trouve sa racine dans notre faculté d'apporter du nouveau dans le monde. En d'autres

\footnotetext{
${ }^{45}$ Pour cette analyse célèbre de la joie, voir « La conscience et la vie », in H. Bergson, La Pensée et le mouvant, op. cit., p. 23 et $s q$.

${ }^{46}$ Idem.
} 
termes, seul un être capable de surprendre peut être surpris. $\mathrm{L}^{\prime}$ «impression de nouveauté » dont parle Bergson au début de son article sur «Le possible et le réel», doit donc s'entendre non seulement à la forme passive d'une affection de notre sensibilité par la nouveauté, mais également, et indissociablement, à la forme active et causale, au sens de la faculté d'imprimer du nouveau dans le monde, autrement dit d'agir librement. La surprise se charge par là d'un sens cosmologique, puisqu'elle apparaît à la pointe de l'évolution comme l'effet consécutif au surgissement d'un être, l'homme, qui « représente une certaine somme de contingence s'introduisant dans le monde $»^{47}$.

Cependant, l'affirmation selon laquelle l'acte libre serait source de surprise ne va nullement de soi. S'il peut en effet paraître évident que la réalité extérieure, telle qu'elle s'offre à ma perception, ne puisse se donner, on l'a vu plus haut, que sous le régime de la surprise (je ne décide pas du contenu de ma visée perceptive), il en va tout autrement de l'acte libre qui, une fois distingué de l'acte fou, gratuit ou arbitraire, est bien la conséquence - c'est même là sa structure typique - d'un motif, d'une résolution ou d'un projet, qui le préparent voire le préfigurent, et dont il est l'actualisation. La définition que Bergson donne de l'acte libre ne rend pas nécessairement plus claire cette corrélation entre surprise et acte libre. D'après lui en effet un acte est d'autant plus libre qu'il exprime davantage le moi profond de son auteur : "Nous sommes libres quand nos actes émanent de notre personnalité tout entière, quand ils l'expriment, quand ils ont avec elle cette indéfinissable ressemblance qu'on trouve parfois entre l'œuvre et l'artiste ${ }^{48}$. N'est-ce pas dès lors, et à l'inverse, l'acte le moins libre qui susciterait le plus de la surprise chez ceux qui nous connaissent, précisément parce que cet acte ne nous ressemble pas? En rattachant la liberté de l'acte à la continuité temporelle de la personne, c'est la possibilité de la surprise qui semble tout d'abord compromise.

Cependant, Bergson invoque le caractère soudain que prennent parfois nos décisions les plus libres. Ainsi, explique-t-il dans un

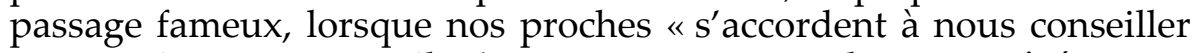
un acte important», "il n'est pas rare que », de notre côté, "une révolte se produise » ${ }^{49}$. Bergson explique : "C'est le moi d'en bas qui remonte à la surface, c'est la croûte extérieure [des conseils] qui

\footnotetext{
${ }^{47} \mathrm{H}$. Bergson, L'Évolution créatrice, op. cit., p. 262.

${ }^{48}$ H. Bergson, Essai, op. cit., p. 129.

49 Ibid., p. 127.
} 
éclate, cède à une irrésistible poussée $»^{50}$. Mais cette discontinuité est en réalité l'effet d'une continuité. La révolte révèle d'un coup la série dynamique de nos états, "sentiments et idées ", qui se succédaient dans les profondeurs de notre conscience. La surprise n'est plus celle suscitée par des forces aveugles et inconscientes de la nature, mais par un acte individuel qui est une initiative.

L'acte libre que Bergson fait surgir dans le troisième chapitre de l'Essai comme un "coup d'État» ou comme un coup de théâtre, comme ce qui surprend tout le monde, n'est donc pas incompatible avec le critère temporel de l'expression de soi, du soi. C'est dans l'instant de son surgissement, que la décision surprend, tandis qu'elle apparaitra rétrospectivement conforme à la personnalité profonde de celui qui s'est décidé. Si l'acte libre est imprévisible, en ce sens qu'il est une création, il n'est pas arbitraire, contrairement à ce qu'on a pu objecter à Bergson. "L'acte libre, dit Bergson, est incommensurable avec l'idée, et sa "rationalité" doit se définir par cette incommensurabilité même, qui permet d'y trouver autant d'intelligibilité qu'on voudra $»^{51}$. Si l'acte libre peut être surprenant et sensé, surprenant sans être gratuit, c'est parce qu'il transcende tout motif, toute raison, parce qu'il n'est pas précédé par tel ou tel principe qui pèserait sur la volonté pour la déterminer. Sa rationalité ou son intelligibilité ne sont pas pour autant absentes, mais rétrospectives, dans la mesure où tel motif, considéré avant que la décision ne soit prise, n'est pas déterminant, mais tout au plus le deviendra sous l'effet et par la vertu de la décision. L'acte libre apparaît donc comme la durée en personne : un processus d'élaboration continue, une succession de moments nouveaux et irréductibles, plutôt qu'un événement.

Lorsqu'elle célèbre 1'action comme ce qui interrompt tout processus nécessaire, Hannah Arendt se montre étonnamment proche de Bergson. Comme lui, elle fait de l'agir la faculté qui en l'homme brise la chaîne de causalité et les enchaînements automatiques, en instaurant quelque chose de neuf et d'inattendu, dans lequel elle va jusqu'à apercevoir un "miracle»: "Le nouveau apparaît toujours comme un miracle. Le fait que l'homme est capable d'action signifie que de sa part on peut s'attendre à l'inattendu, qu'il est en mesure $\mathrm{d}^{\prime}$ accomplir ce qui est infiniment improbable $»^{52}$. Le terme " miracle » n'est pas étranger à Bergson, qui voit dans l'acte libre la manifestation d'une force qualifiée de miraculeuse, la volonté, capable de donner

\footnotetext{
50 Idem.

${ }^{51}$ H. Bergson, L'Évolution créatrice, op. cit., p. 48.

${ }^{5}$ H. Arendt, Condition de l'homme moderne, Paris, Pocket, « Agora », 2013, p. 234.
} 
plus qu'elle n'a: «La volonté est en effet quelque chose de merveilleux, quelque chose qui par certains côtés, par certains aspects au moins, participe du miracle. La volonté est bien une force qui est capable de $s^{\prime}$ accroître elle-même indéfiniment $»^{53}$. En apportant quelque chose de nouveau dans le monde, la volonté apparaît à Bergson comme le déploiement dans l'univers d'une force d'un genre spécial, non pas physique, mais psychique, qui déjoue toute tentative de prévision, surprenante par essence, puisque les conditions d'équivalence des causes et des effets ne seraient pas remplies dans son cas. L'existence d'une telle force, échappant au principe de la conservation de l'énergie, ne remet pas cependant en cause les lois de la science, mais elle oblige à supposer que les systèmes conservatifs, dont les éléments sont susceptibles après transformation de revenir à leur position initiale, ne sont les seuls possibles.

Ce rapprochement entre Arendt et Bergson n'est pas purement extérieur et arbitraire. Dans la deuxième partie de La Vie de l'esprit consacrée au "Vouloir", et dans un chapitre intitulé "Le problème du nouveau », Arendt fait l'éloge de la riposte de Bergson à ce que l'on pourrait appeler l'oubli de la nouveauté dans la philosophie, et elle se montre particulièrement sensible à la compréhension de l'acte libre qui circule dans toute son œuvre. Elle cite à ce propos la fin de l'article de Bergson sur «Le possible et le réel »: «La plupart des philosophes n'arrivent pas, quoi qu'ils fassent, à se représenter la nouveauté radicale et l'imprévisibilité... D'une action entièrement neuve... ils semblent ne se faire aucune idée ${ }^{54}$. Parce qu'ils sont agissants les hommes ont la faculté, selon Arendt, très proche de Bergson sur ce point, d'apporter quelque chose de nouveau, ce qui signifie que leur action transcende aussi bien les motifs, les raisons, les buts ou les intérêts. Entre les deux penseurs, les différences seraient certes nombreuses et radicales, à commencer par la dimension intrinsèquement politique de l'action chez Arendt, perspective très éloignée des conditions de l'acte libre chez Bergson. On aurait tort pourtant de réduire l'action chez Arendt à cette seule dimension politique pourtant privilégiée par elle. Toute son œuvre est soustendue par une ontologie de la nouveauté qui mériterait d'être confrontée à celle de Bergson, avec laquelle elle partage de concevoir l'action comme ce qui introduit dans le monde une part irréductible

\footnotetext{
53 Bergson, cours du Collège de France sur «L'Évolution du problème de la liberté », daté du 10 mars 1905, édité par A. François et reproduit dans Le magazine littéraire, n 468, octobre 2007, p. 86.

${ }^{54}$ H. Arendt, La Vie de l'esprit, Paris, PUF, «Quadrige », 1981, p. 315 ; les coupures dans la citation sont d'Arendt.
} 
d'imprévisibilité et de surprise. Cette exigence commune les a d'ailleurs exposés aux mêmes malentendus et aux mêmes accusations d'irrationalisme. Or, pour l'un comme pour l'autre, la nouveauté ne compromet pas toute rationalité, mais seulement celle, étroite, qui rabattrait toute compréhension, et notamment en histoire chez Arend $\mathrm{t}^{55}$, sur des logiques causales et déterministes, et celle-ci reprend explicitement à Bergson sa critique de l'illusion rétrospective, lorsqu'elle dénonce l'usage de la causalité dans les sciences historique qui laisse croire en l'existence d'enchaînements nécessaires qui ont conduit au surgissement de tel ou tel événement. Selon Arendt au contraire, l'événement, imprévisible et neuf, surgit toujours comme une surprise, et $c^{\prime}$ est lui qui "éclaire son propre passé » ${ }^{56}$, sans pouvoir jamais en être déduit. Mais cette surprise, du côté de l'historien et des témoins, est inséparable de la capacité de surprendre que possèdent les hommes, et qui correspond en eux à la faculté d'agir, autrement dit, pour Arendt, à celle de commencer, qui s'enracine elle-même dans notre propre commencement, c'est-à-dire dans notre naissance, qui n'est pas chez elle seulement un événement, mais une faculté. En d'autres termes, qui conviendraient aussi bien à Bergson, la surprise devant la nouveauté est inséparable de la capacité d'innover. Arendt explique ainsi que lorsque la liberté surgit dans l'Histoire sous la forme de la nouveauté révolutionnaire (pendant politique en quelque sorte de la révolte dans l'histoire de l'individu chez Bergson), la surprise que suscite son surgissement, s'enracine dans l'aptitude humaine à commencer. De même pour Bergson, la surprise que nous éprouvons devant la nouveauté dans l'univers, celle de la vie ou de la nature, est comme l'écho, à l'extérieur de nous, de ce dont nous sommes capables à travers nos actes libres. Et c'est pourquoi Bergson ne cesse d'éclairer l'extérieur par l'intérieur, l'impression de nouveauté par la production du nouveau.

Si Bergson revient constamment à l'acte libre pour appréhender la nouveauté, si même il part de lui dans son premier livre, qui articule si fortement les dimensions du temps et de la liberté, c'est parce que

\footnotetext{
${ }^{55} \mathrm{H}$. Arendt ne cesse de revenir sur cette illusion dans des considérations d'épistémologie de l'histoire qui jalonnent une grande partie de son œuvre, de même que Bergson d'ailleurs, qui ne s'en tient pas à la critique de cette illusion dans les conceptions de la vie et de la liberté, mais en dénonce les effets dans la connaissance historique elle-même, dans la première partie de l'introduction à La Pensée et le mouvant.

${ }^{56} \mathrm{H}$. Arendt, La nature du totalitarisme, Paris, Payot, 1990, p. 61. Sur la question de l'événement et de la surprise chez Arendt, voir l'étude d'É. Tassin, «Événements versus bifurcations : digressions philosophiques sur la raison des miracles", in Bifurcations: les sciences sociales face aux ruptures et à l'événement, M. Bessin (dir.), Paris, La Découverte, 2010.
} 
la nouveauté, qui est le sens fondamental de la durée, depuis la sensation la plus simple à la décision la plus grave, n'est jamais pour nous l'objet d'une visée ou d'une vision, un pur spectacle, mais l'attestation d'un acte de notre conscience par lequel elle fait tenir ensemble les moments de notre expérience pour les prolonger les uns dans les autres, faute de quoi il n'y aurait qu'un simple clignotement, une pure répétition du même. Durer est donc une activité ! Tel est ce qu'on pourrait apercevoir si l'on pouvait se regarder agir. Mais pour apercevoir cet acte, il faudrait produire un effort, et même se faire violence, car le regard est naturellement spatialisant. Il reste que ce serait là le modèle de l'intuition c'est-à-dire d'une connaissance du réel qui ne le modifierait pas, mais qui le saisirait en train de se faire. Il faudrait se tenir en ce point ou sur cette pointe, où coïncideraient « voir » et «agir », afin que s'exerce un « voir » immanent à l' « agir » ou au « vouloir». Telle est cette contorsion à laquelle notre conscience devrait se plier: "Il faudrait qu'elle se détachât du tout fait et s'attachât au se faisant. Il faudrait que se retournant et se tordant sur elle-même, la faculté de voir ne fît plus qu'un avec l'acte de vouloir $»^{57}$. Ou en $d^{\prime}$ autres termes, très proches :

Essayons de voir, non plus avec les yeux de la seule intelligence, qui ne saisit que le tout fait et qui regarde du dehors, mais avec l'esprit, je veux dire avec cette faculté de voir qui est immanente à la faculté d'agir et qui jaillit, en quelque sorte, de la torsion du vouloir sur luimême ${ }^{58}$.

C'est en ce point précis que la surprise pourrait éclairer l'intuition de la durée, elle-même envisagée à partir de l'acte libre. Saisir quoi que ce soit dans sa réalité, c'est-à-dire dans sa temporalité, ce sera, pour Bergson, le saisir sur le même mode que l'acte libre, c'est-à-dire le surprendre dans le cours de son processus, sur le vif, en train de se faire. Tout autre manière de $s^{\prime} y$ prendre pour connaître quelque chose, en cherchant par exemple à le décrire ou à l'expliquer, reviendra à en manquer la réalité. L'acte libre est un cas singulier de durée qui permettrait de mettre au jour la structure si paradoxale de l'intuition dès lors qu'on l'envisage comme l'acte de surprendre, dont les deux sens, de l'acte imprévisible et de la connaissance participative, fusionneraient, à la limite, en un être capable de se surprendre lui-même.

${ }^{57}$ H. Bergson, L'Évolution créatrice, op. cit., p. 238.

58 Ibid., p. 251. 
